\title{
The Criticism and Amendment for the Dual-Factor Model of Mental Health: From Chinese Psychological Suzhi Research Perspectives
}

\author{
Xinqiang Wang, Dajun Zhang* \\ Key Laboratory of Cognition and Personality of Ministry of Education, Center for Mental Health Education, Southwest University, \\ Chongqing, China. \\ Email: zhangdj@swu.edu.cn, xinqiang.wang@hotmail.com
}

Received May $5^{\text {th }}, 2012$; revised June $17^{\text {th }}, 2012$; accepted July $20^{\text {th }}$, 2012

\begin{abstract}
Aiming at the limitations of psychopathology (PTH), the dual-factor model of mental health (DFM) was proposed as a new mental health concept and methodology under the background of positive psychology trend. In this paper we propose giving an overview of DFM, and doubt, criticize, and modify DFM from the perspective of Chinese psychological suzhi research. The available literature from 1983 to 2012 that is related to DFM and concerning psychological suzhi research in the past 20 years has been reviewed. In addition, we also absorbed the idea of positive psychology and Traditional Chinese Medicine (TCM) Constitution theory to develop theoretically the relationship model between psychological suzhi and mental health. The relationship model between psychological suzhi and mental health modifies and transcends PTH and DFM. It will be the new research area of mental health research.
\end{abstract}

Keywords: Dual-Factor Model of Mental Health; Psychopathology; Relationship Model between Psychological Suzhi and Mental Health; Subjective Well-Being

\section{Introduction}

The fourth edition of the Diagnostic and Statistical Manual of Mental Disorders (DSM), DSM-IV has been issued by the American Psychiatric Association since all countries are paying increasing attention to human beings' mental health. The DSM-IV includes the diagnostic standards and therapeutic plans for more than 340 kinds of mental or psychopathologic illness, but it does not actually explain ways to relieve the pain for patients of psychological illnesses. Meanwhile, the number of psychological patients across the world has increased [1]. Therefore, we should reflect on the traditional mental health model and strive to find a new and scientific mental health model with high effectiveness and efficiency. The dual-factor model of mental health (DFM) was set forth under the background of positive psychology to solve the deficiencies of the traditional model of mental health [1-7].

\section{Basic Concepts of Dual-Factor Model of Mental Health}

\subsection{It Insists That Mental Health Should Be a Complete State}

The DFM insists that mental health is not the absence of

\footnotetext{
"Corresponding author.
}

mental illness or the high subjective well-being (SWB), but a complete state that integrates the absence of mental illness and the high SWB. Well-being and mental illness are two related, but distinct, dimensions of mental health $[2-4,8]$. This corresponds with the opinions of Headey [9]: Well-being (life satisfaction, positive affects) and ill-being (anxiety, depression).

\subsection{It Classifies the Mentally Healthy People}

The DFM takes SWB, the positive indicator, and psychopathology (PTH) symptoms, the negative indicator as two indispensable factors for mental health diagnosis. It can be used to classify people into complete mental health, incomplete mental health, incomplete mental illness and complete mental illness four groups based on such indicators and to forecast the mental health functions of those four groups and the development trend of their mental health $[1,3,6,7]$.

\subsection{Purpose of Psychological Prevention and Intervention}

Previous research showed that individuals' mental disorder in the early stage may induce other complications, increase the probability of relapse of mental illness and 
make the treatment more complicated [1]. Therefore, the DFM emphasizes positive prevention and advocates the use of the aforesaid two-dimensional (PTH and SWB) classification standard to identify which individual needs to improve the SWB in order to actively prevent mental illness and which individual need intervention [3,7] "Symptom reduction may be only a first step in treatment” (pp. 50-51) [1]. This model no longer makes patients remain in incomplete mental health (languishing) and deems disappearance of syndromes as the end of treatment, but holds that incomplete mental health may be the intermediate point for individuals to suffer mental illness or achieve complete mental health. It insists that intervention and treatment should help people overstep the baseline of their previous psychological functions and finally achieve complete mental health. It believes that the enhancement of such positive factors as SWB will improve the effects of intervention and treatment and complete mental health may effectively reduce the probability of relapse [1,7].

\section{Deficiencies of Dual-Factor Model of Mental Health}

Firstly, it is about the value and necessity of SWB intervention. Some existing research has acknowledged that SWB is consistent across situations and is stable across the life span, even after the occurrence of intervening life events [10]. Some recent researches also indicated that some events are so tragic (e.g. the death of one's child, and divorce) that people never regain their set-point, and that there are deficiencies in the set point theory of well-being, individuals may have a set point for SWB, around which SWB rises and falls over time but to which SWB eventually returns $[9,11]$. It indicates that although positive emotion or life satisfaction is relatively easier to be improved in a short time, they are not stable after training or development. The duration is short. Around the set point, they may fluctuate. The intervention gets half the result with twice the effort, or is even futile. Therefore, its value as an effective indicator is greatly reduced. The scientific effectiveness of overstressing and partially focusing on strengthening SWB is doubtful [12]. SWB indicator as the tool to evaluate the current positive state of individuals may be effective and necessary, which corresponds to the focus of DFM. However, we believe that to understand the state of mental health (positive or negative) is just the beginning of diagnosis. It is important for prevention, facilitation or treatment, but it is far from sufficient for fundamentally preventing, maintaining and facilitating mental health and for thoroughly removing mental disorders. It is because that to reach and maintain the state of complete mental health as stated by DFM, the moderating or mediating factor (the factor can be effectively intervened or cultivated, and is stable to a certain extent) behind the current state of mental health, as well as the mechanism of action should be further studied.

Secondly, do SWB and PHT share a cause-effect relationship, or are the results related? The World Health Organization's conceptualization of mental health as a positive state of psychological well-being goes beyond the absence of disease [13]; namely, no matter positive mental health or negative mental health, they are substantially a mental state [14], and are consequential psychological states under life events. Therefore, SWB may not be the factor that influences mental illness. SWB is not predictable. SWB may only be related to mental illness and is also a consequence of mental health state. People may question the mentioned opinions with the fact that "the improvement of SWB eases depression, anxiety and other mental illnesses". Now, it is time to introduce the third point.

Thirdly, can mental health state be improved through education, or can it be moderated, maintained or facilitated through a third party? Hai-Zhong Zhang believed that "mental health cannot be improved directly through education" (p. 59), and "'state' cannot be improved directly through education. Education improves the suzhi (namely quality) of people through knowledge conveyance and behavioral training” (p. 59) [15]. Educators have gradually realized that mental health not only has external reflections (psychological state), but also has substantial characteristics (psychological suzhi). For example, researchers believed that "good adaptability and behaviors are external reflections, and the substance is good psychological suzhi” [16]. Da-Jun Zhang believed that "the psychological suzhi of the youth and children is the core of their psychological structure, and the substance of psychological activities (domination role); and mental health is the state layer (surface layer or explicit layer) of psychological structure, and also the reflection of the state (symptom) of psychological suzhi. Therefore, in terms of improving the psychological suzhi of the youth and children, the basic countermeasure or the fundamental way to solve the mental problems of the youth and children is to foster sound psychological suzhi of the youth and children" (p. 27) [17]. Which one is the cause? And which one is the effect? Obviously, it is long-term (rather than short-term) state that forms the psychological suzhi of individuals, and the psychological suzhi of individuals dominate the current state of individuals $[12,14]$. Peterson and his colleagues carried out a retrospective study through sampling on the Internet. And the results showed that character strengths (the definition of character strengths overlaps and is similar the definition of psychological suzhi) help individuals to 
recover from physical and mental illnesses. Thus, researchers inferred that the intervention measures that facilitate such character strengths will help those who suffer from physical or mental health crisis to better recover physical and mental health [18]. Therefore, mental health is a state and cannot be improved directly through education [12]. Education and intervention can only improve psychological suzhi or character strengths and other psychological factors. Behind the result that the improvement of SWB reduces depression, anxiety or other mental illnesses is the mediating or moderating effects of psychological suzhi, which is a necessary basis for setting forth the relationship model between psychological suzhi and mental health.

\section{Preliminary Development of Relationship Model between Psychological Suzhi and Mental Health}

Researchers believe that mental health should include more positive assets, strengths, resilience and other indicators of wellness. Although the DFM does not focus on the evaluation of strengths, it paves the way for strengthbased assessments [19]. According to DFM, specific fundamental individual characteristics, such as personality, may substantially influence children's mental health status [6]. These point out the direction for "predicting mental health based on psychological suzhi" or "fostering psychological suzhi to promote and substantially maintain mental health" [14]; and reflect the thought that psychological suzhi should be included in the model to develop relationship model between psychological suzhi and mental health.

\subsection{Connotation and Structure of Psychological Suzhi}

Psychological suzhi is a concept set forth by Chinese scholars under the background of quality-oriented education, and by absorbing the essence of traditional culture $[14,20]$. Psychological suzhi is an important, integral part of people's characteristics [20]. As a Chinese-originated, there is no corresponding conception in the Western psychology literature for psychological sushi [21,22]. To help readers have a better understanding about this conception, Zhang coined the word psychological suzhi, in order to avoid reader's confusion [21]. In Chinese psychology, this conception had also been translated as mental quality, psychological quality, mental diathesis, or psychological diathesis. However, the word "quality" cannot cover the whole meaning of suzhi [22]. In recent decades, many Chinese scholars including Dajun Zhang, Jisheng Wang, Deli Shen, Guocai Yan, Zhengzhi Feng have been conducting a lot research on psychological suzhi. From 1980 to 2011, in the China Academic Jour- nal Network Publishing Database (CAJD), the title contains “心理素质” (psychological suzhi in Chinese) academic papers have 4990, keywords contains “心理素质” academic papers have 31293, only 2011 this year keywords contains “心理素质” academic papers also have 2267. Among them, the Psychological Suzhi Research Team of the Southwest University led by Zhang Da-Jun is the most influential and the most recognized team [23].

Based on individuals' physiological conditions, psychological suzhi is a steady, fundamental and derivative mental quality or characteristic, which is formed through the internalization of extrinsic elements and is closely related with individuals' adaptive and creative behaviors $[21,24]$. Substantially, psychological suzhi is a kind of psychological quality that consists of three dimensions, including cognition quality, personality quality, and adaptability. These three dimensions can be further divided into 22 elements, such as comprehensiveness, swiftness, openness, purposiveness, inquisitiveness, persistence, awareness, monitoring, independence, optimism, emotional stability, adventurousness, interpersonal adaptation, stress adaptation, etc [24], and are evaluated through typical behaviors [14]. In terms of structure-function, Da-Jun Zhang also believed that psychological suzhi is the integration of elements and functional value of psychology and behaviors [25]. Elements include cognition quality and personality quality, and functional value is the adaptability developed based on elements [14]. To be specific, cognition quality is indicated by human's reflection of the objective reality. Cognition quality is directly involved in the cognition of the objective reality, and is the basic component of psychological suzhi. Personality quality is reflected by how people treat the objective reality. Although personality quality cannot directly cognize the objective reality, it motivates and regulates cognition. Personality quality is the motivetional component of the elements of psychological suzhi. Adaptability is the habitual behavior tendency reflecting based on the cognition quality and personality quality, by learning, responding to and defending the external social environment; by controlling, understanding and adjusting the internal psychological process; and through the interaction with the social and living environment. It is a factor in the structure of psychological suzhi that has the most derivative functions. Therefore, according to the summary and conclusion in terms of structure-function, the model of static relation structure theory of psychological suzhi is showed below (see Figure 1).

\subsection{The Theoretical Basis for the Relationship Model between Psychological Suzhi and Mental Health}

Substantially, psychological suzhi is a stable psycho- 


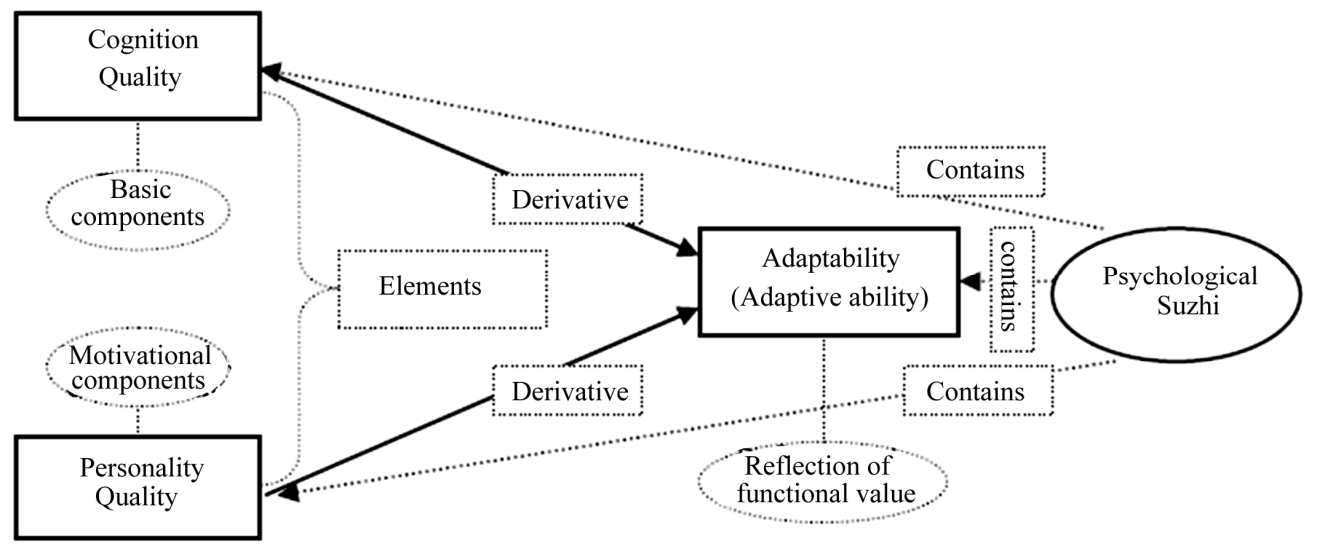

Figure 1. Model of relation structure theory of psychological suzhi.

logical quality, which includes "multi-level self-organizing system from stable endogenesis psychological quality to explicit behavioral habits" [26]; while mental health is a positive and good psychological state. Da-Jun Zhang set forth that "generally speaking, those who have sound or high psychological suzhi will seldom have mental disorders; even if psychological problems occur, they can usually adjust everything by themselves; normally they have healthy psychology. On the contrary, those who have unsound or low psychological suzhi will have mental disorders easily; or faced with psychological problems, they cannot adjust easily by themselves; and normally they have unhealthy psychology" [17]. Psychological suzhi moderates or plays a mediation role in how the external risk and pathogenic factors or protective factors influence mental health, for example, psychological suzhi moderates and plays a mediation role in the protection provided by social support system to mental health. Many research into the relationship between mental health and subjective support, objective support \& utilization of support showed that "for mental health, the most important aspect is that whether people feel they have a sufficient and good social support system, and whether they can utilize the system effectively, rather than how many helps the system actually has provided" (p. 458) [27]. In addition, psychological suzhi also plays a decisive role in the self-regulation and self-recovery of individuals. Substantially, the relationship between psychological suzhi and mental health is "root cause" and "symptom", which is similar to the relationship between constitution and health state.

As a conception of Traditional Chinese Medicine (TCM) Constitution Theory, constitution is the integration of specific physical qualities and certain psychological qualities [28]. It refers to the comprehensive and relatively stable qualities or individual characteristics of morphological structure, physiological functions and psychological states that are developed based on innate gift and acquisition during the life process of people $[28,29]$. In many cases, constitution determines the vulnerability to some disease and the tendency during pathological changes, and influences the formation of "symptoms". Constitution restricts the changes and outcomes of "symptoms", namely constitution is the basis for "different symptoms for the same disease" and "the same symptom for different diseases". The research by He Wen-Bin and He Ling into the Yellow Emperor's Canon of Medicine illustrated the theory well: "the Yellow Emperor's Canon of Medicine focuses on individual characteristic, constitution and other internal factors; and believes that even with the same emotion and stimuli of psychological and social factors, some will be sick while the other not, and the location of illness and symptoms will be different, the root cause for which is the differences of individual characteristics and constitution. Thus treatment should be based on individual characteristic and constitution" [30]. It also explains "why individuals with different psychological suzhi will have different mental health states when they face the same risk factors, namely, factors can modify individual responsiveness to stress”.

Researchers believed that "Application of a general immunity model as a common framework to resilience research in mental health can help to clarify underlying mechanisms and challenges, which contribute to our understanding of health in general and mental health in particular" (p. 491) [31]. Resilience refers to individual's capacity and traits to respond to stress, frustration, trauma and negative events [32]. It can be studied from individual power and supportive power [33-35]. The connotation of individual capacity partially overlaps that of psychological suzhi, and there are similarities between them [34,35], such as optimism. The difference lies in that psychological suzhi is a kind of behavior that is reflected in behaviors and activities, and that psychological suzhi emphasizes the relatively stable psychological 
quality and individual power. Although psychological suzhi is different from resilience, they both highlight the core role of individual quality or capacity in mental health. Resilience research from the view of individual quality or capacity also provides important theoretical and proof basis for development of the relationship model between psychological suzhi and mental health. In addition, the research and theory of the relationship between personality traits and mental health, and the research and theory of the relationship between character strengths and mental health also provide important theoretical and proof basis, which has been illustrated in detail in other papers, and will not be illustrated in the this article.

\subsection{Basic Theoretical Views of the Relationship Model between Psychological Suzhi and Mental Health}

Existing researches have proved that mental health is the problem of individuals and also the problem of groups and the society [36]. From the perspective of individual mental health, the model focuses on individual psychological suzhi and individual psychological quality; considers the important role of social environment and support system in an individual's mental health; theoretically develops the relationship model between psychological suzhi and mental health based on existing research results and theoretical analysis. The connotation of the model mainly includes two related levels.

\subsubsection{The Mechanism Level}

The core concept of the model is that the external risk factors and protective factors act through psychological suzhi, and emphasizes that psychological suzhi is the key of mental health problem. This concept has been recognized by a large number of psychologists and official documents of the government in China. For example Chong-De Lin holds that "the key of mental health education lies in improving the psychological suzhi of students" [37]; Da-Jun Zhang believes that "the fundamental means to maintain the mental health of students is to foster sound psychological suzhi” (p. 494) [26]; HaiZhong Zhang argued that "psychological suzhi must be intervened to improve mental health" [15]; according to the Outline of Guidance for Mental Health Education of Pupils and Middle School Students (September 25, 2002) issued by Chinese ministry of education, "promote the development of healthy psychological Suzhi, and maintain mental health of students", and "gradually im- prove their psychological suzhi”. The mechanism level includes the sub-mechanism levels for the development of psychological suzhi and action of psychological suzhi.

\subsubsection{Sub-Mechanism Level for the Development of} Psychological Suzhi

It sets forth that psychological suzhi is developed during the interaction of social and cultural environment and hereditary and physiological basis when external stimuli is gradually internalized into stable, basic and implicit psychological suzhi [24]. Such psychological quality is relatively stable, which will further cause the initiative selection of social and cultural environment, goes after profits and avoids disadvantages, so that it will selectively accept the influence of the environment and maintain or promote the development or improvement of psychological suzhi (see Figure 2).

\subsubsection{Sub-Mechanical Level for the Action of Psychological Suzhi}

It sets forth that individual psychological suzhi plays a crucial moderating or mediating role in the occurrence and development of illness, and that psychological suzhi and mental health state (including positive mental health state and negative mental health state) influences each other and develop dynamically. To be specific, it is reflected by that: the external risk factors of mental problems act through psychological suzhi (internal); the cognition quality and personality quality are the endogenous factors for the occurrence and development of mental problems; adaptability in the structure of psychological suzhi reflects the interaction between external and internal causes, and is the intermediary step for the action of external cause through internal cause; the level and the sound degree of psychological suzhi moderates and plays an mediation role in how the external risk factors influence an individual's mental health state; maintaining a certain state of the individual's mental health that further affects and reshapes individual psychological suzhi (see Figure 2).

With respect to the influence of external environment and psychological suzhi on an individual's mental health, if the influence of one of them increases, the influence of the other one will decrease. And the proportion of their influence is related with the age of individuals (see Figure 3). Generally speaking, the influence of psychologycal suzhi will increase when people are growing up, and will decrease slightly when they become old. On the contrary, the influence of external factors on mental health will decrease as people are growing up and will slightly increase when they become old. Therefore, attention to and research into the action mechanism of psychological suzhi for adolescence, and strengthening psychological suzhi training or cultivating of adolescence are of significant practical and theoretical meanings to the adolescence who rebel parents' protection, and who are going through fast but unstable physical and psycho- 


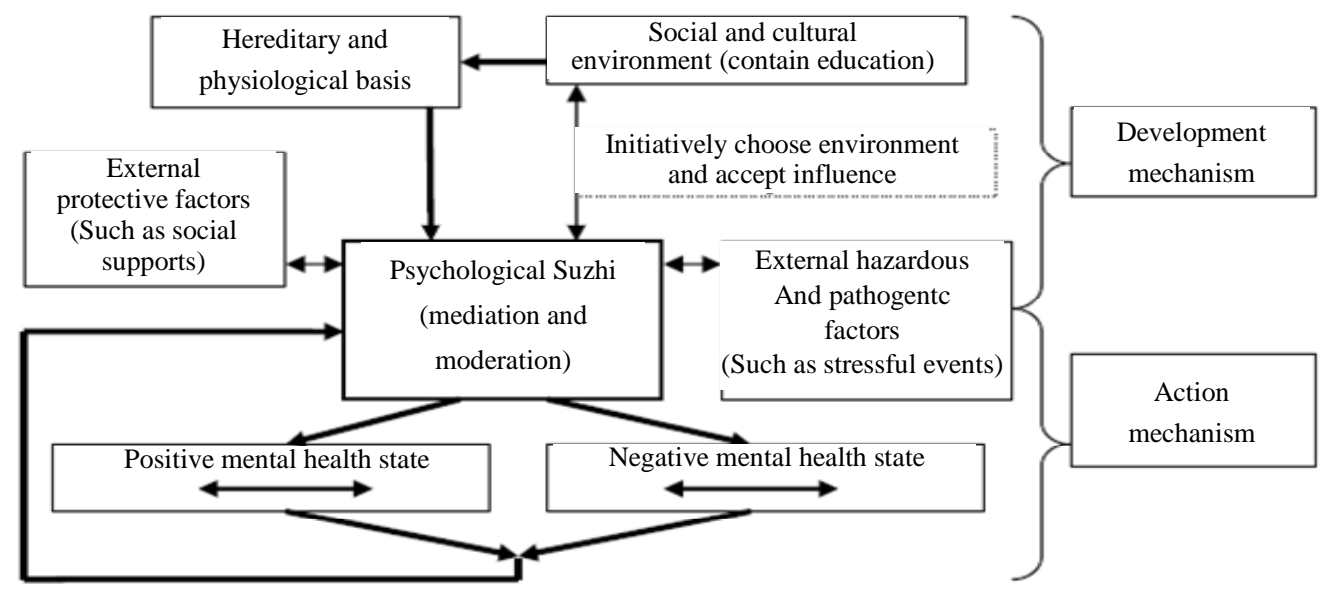

Figure 2. Theoretical model of mental health mechanism of the relationship model between psychological suzhi and mental health.

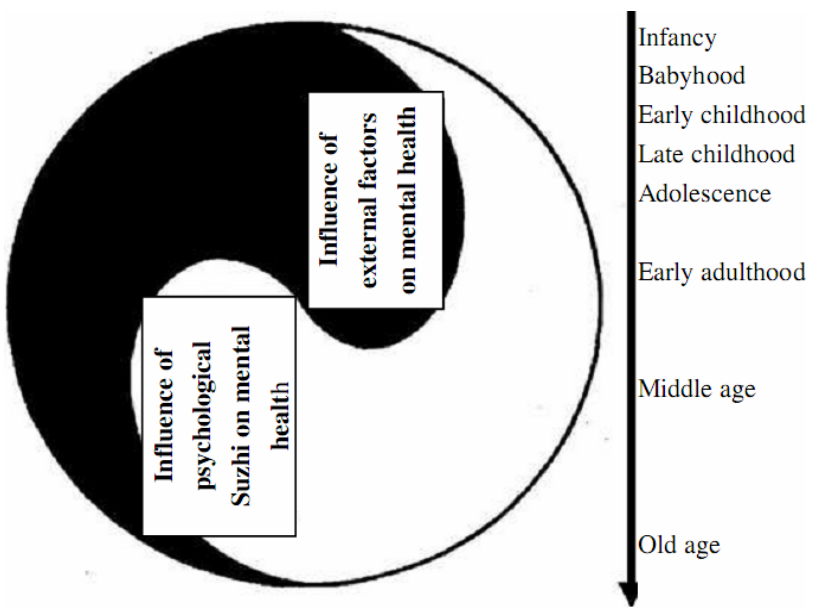

Figure 3. The influence of external environment and psychological suzhi on an individual's mental health.

logical changes, and who have troubles related to mental health frequently.

\subsubsection{Assessment and Target Level}

With respect to the assessment and diagnosis of current state of an individual's mental health and overall situation of the individual's mental health, the relationship model between psychological suzhi and mental health sets forth that the classified and qualitative assessment should be combined with quantitative assessment; namely, assess the individual's situation on a classified and on qualitative basis, and then based on the assessment results, perform quantitative assessment.

\subsubsection{Classified and Qualitative Assessment}

The assessment includes the classified assessment of the current state of mental health, and assessment of the overall situation of an individual's current mental health.

Classified assessment of the current state of the indi- vidual's mental health is to assess the current state of the individual's mental health by combining the score of positive mental health indicator (such as SWB) with score of negative mental health indicator (such as depression). And then classifies individuals into four categories, namely, complete mental health, incomplete mental health, incomplete mental illness and complete mental illness, which are the same as DFM classification $[1-3,6,7]$.

The assessment of the overall situation of current mental health is based on the score of positive mental health indicator, score of negative mental health indicator (Score is obtained by reverse-scoring of negative indicator), and the score of psychological suzhi indicator. If only the score of psychological suzhi indicator is lower than cut point, then the individual is classified into Vulnerable I (mild) group; if the score of psychological suzhi indicator and score of positive mental health indicator are lower than cut point, then the individual is classified into Vulnerable II (severe) group; if the score of psychologycal suzhi indicator and score of negative mental health indicator are lower than cut point, then the individual is classified into Troubled I (mild) group; if the score of positive mental health indicator, score of negative mental health indicator, and the score of psychological suzhi indicator are all lower than cut point, the individual is classified into Troubled II (severe) group; if only the score of positive mental health indicator is lower than cut point, the individual is classified into Easy Recovery I (mild) group; if only the score of negative mental health indicator is lower than cut point, the individual is classified into Easy Recovery II (moderate) group; if the score of positive mental health indicator, and score of negative mental health indicator are both lower than cut point, the individual is classified into Easy Recovery III (severe) group; if no score is lower than cut point, the individual is classified into Flourishing group (see Table 1). 
Table1. Grouping and grouping standard of the relationship model between psychological suzhi and mental health.

\begin{tabular}{ccccl}
\hline & $\begin{array}{c}\text { The score of psychological } \\
\text { suzhi indicator lower } \\
\text { than cut point }\end{array}$ & $\begin{array}{c}\text { The score of positive mental } \\
\text { health indicator lower } \\
\text { than cut point }\end{array}$ & $\begin{array}{c}\text { The score of negative mental } \\
\text { health indicator lower } \\
\text { than cut point }\end{array}$ & Category \\
\hline YES & NO & NO & Vulnerable I (mild) group \\
Lower than \\
cut point?
\end{tabular}

Note: The score of negative mental health indicator is obtained by reverse-scoring of negative indicator.

\subsubsection{Quantitative Assessment}

The assessment also includes the assessment of the current state of mental health, and assessment of the overall situation of individual's current mental health.

The State Assessment Index (SI) is the product of the standard score of positive mental health indicator (PI, such as SWB) and the standard score of negative mental health indicator (NI, such as PHT) (Score is obtained by reverse-scoring of negative indicator), which is the underside area as shown in Figure 4.

The Overall Assessment Index (OI) is the products of the score of positive mental health indicator(PI), score of negative mental health indicator(NI), and the score of psychological suzhi indicator (SZI), namely $\mathrm{OI}=\mathrm{PI} \times \mathrm{NI}$ $\times$ SZI, and is the volume shown in Figure 4 .

\subsubsection{Application Methods and Intervention Tips for Classified and Qualitative Assessment and Quantitative Assessment}

The relationship model between psychological suzhi and mental health is a methodology model. Researchers can adopt overall measurement (namely measurement of PHT, SWB and overall psychological suzhi) to classify individuals, diagnose and develop intervention plans. However, what is more important is that specific measurement indicators (such as depression indicator of negative mental health, life satisfaction indicator of positive mental health, and emotional competency indicator of psychological suzhi) should be selected as per the actual needs and the model, so as to classify and diagnose individuals and develop intervention plan.

With respect to the application of qualitative assessment and quantitative assessment, individuals should be classified first according to the score of negative mental health indicator, positive mental health indicator, and psychological suzhi indicator; and then based on such classification, consider the score of current state of mental health, and score of overall situation of individuals' mental health under the same category. Substantial dif-

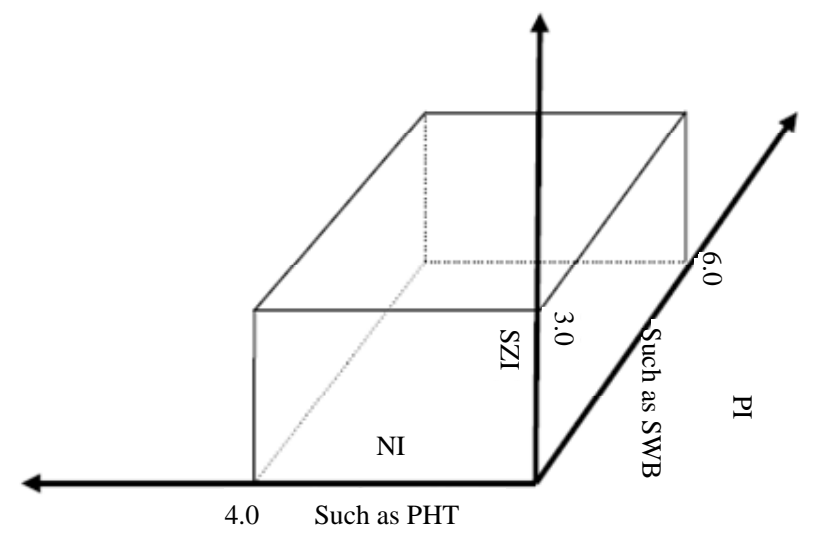

Note: PI is positive mental health indicator; NI is negative mental health indicator; SZI is psychological suzhi indicator.

Figure 4. Simulated diagram of the current state of an individual's mental health and overall situation of mental health under the relationship model between psychological suzhi and mental health.

ference exists among groups, but these groups can be changed through intervention and the relevant factors. SI and OI cannot be compared between different categories, but only under the same category. With higher score, the current state of mental health or the overall situation of mental health will be better.

The relationship model between psychological suzhi and mental health highlights positive prevention of illness; improves psychological suzhi so as to treat mental illness, prevents unstable treatment effect and relapse; and improves psychological suzhi to prevent harm caused by risk and pathogenic factors, and prevents mental illness. Meanwhile, according to the principle of solving both the symptoms and root causes, the model stresses that proper preventive and intervening measures should be arranged as per the respective categories of individuals. The key to cure mental illness, promote and maintain mental health lies in the development of sound psychological suzhi. The eventual target of intervention 
is to realize Flourishing group.

\section{Conclusion}

Although it is not possible to foster psychological suzhi in a short period, psychological suzhi that has been developed or cultivated is relatively stable for a certain period, has the function of forecasting, and is significant for an individual's mental health, academic success, and work performance. Therefore, we believe that the relationship model between psychological suzhi and mental health can effectively overcome the deficiencies of PTH and DFM. Enhancement of psychological suzhi under the model will be a new field of research on mental health and mental health education.

\section{Acknowledgements}

This study was supported by 2010 Key Project "Research for the dual-factor model of mental health in youth" at Key Humanity Social Science Research Institute in Chongqing, China.

\section{REFERENCES}

[1] C. L. M. Keyes and S. J. Lopez, "Toward a Science of Mental Health: Positive Directions in Diagnosis and Interventions," In: C. R. Snyder and S. J. Lopez, Eds., Handbook of Positive Psychology, Oxford University Press, New York, 2005, pp. 45-59.

[2] P. J. Greenspoon and D. H. Saklofske, "Toward an Integration of Subjective Well-Being and Psychopathology," Social Indicators Research, Vol. 54, No. 1, 2001, pp. 81108. doi:10.1023/A:1007219227883

[3] S. M. Suldo and E. J. Shaffer, "Looking beyond Psychopathology: The Dual-Factor Model of Mental Health in Youth,” School Psychology Review, Vol. 37, No. 1, 2008, pp. 52-68.

[4] B. Doll, "The Dual-Factor Model of Mental Health in Youth,” School Psychology Review, Vol. 37, No. 1, 2008, pp. 69-73.

[5] C. L. M. Keyes, "The Mental Health Continuum: From Languishing to Flourishing in Life," Journal of Health and Social Behavior, Vol. 43, 2002, pp. 207-222. doi:10.2307/3090197

[6] S. P. Antaramian, E. S. Huebner, K. J. Hills and R. F. Valois, "A Dual-Factor Model of Mental Health: Toward a More Comprehensive Understanding of Youth Functioning,” American Journal of Orthopsychiatry, Vol. 80, No. 4, 2010, pp. 462-472.

[7] X. Q. Wang, D. J. Zhang and J. L. Wang, "Dual-Factor Model of Mental Health: Surpass the Traditional Mental Health Model," Psychology, Vol. 2, No. 8, 2011, pp. 767-772. doi:10.4236/psych.2011.28117

[8] R. B. Wilkinson and W. A. Walford, "The Measurement of Adolescent Psychological Health: One or Two Dimensions?” Journal of Youth and Adolescence, Vol. 27, 1998, pp. 443-455. doi:10.1023/A:1022848001938

[9] B. Headey, "The Set Point Theory of Well-Being Has Serious Flaws: On the Eve of a Scientific Revolution?” Social Indicators Research, Vol. 97, No. 1, 2010, pp. 7-21. doi:10.1007/s11205-009-9559-X

[10] E. Diener and R. E. Lucas, "Personality and Subjective Well-Being,” In: D. Kahneman, E. Diener and N. Schwarz, Eds., Foundations of Hedonic Psychology: Scientific Perspectives on Enjoyment and Suffering, Chapter 11, Russell Sage Foundation, New York,1999.

[11] R. E. Lucas, P. S. Dyrenforth and E. Diener, "Four Myths about Subjective Well-Being," Social and Personality Psychology Compass, Vol. 2, No. 5, 2008, pp. 2001-2015. doi:10.1111/j.1751-9004.2008.00140.x

[12] X. Q. Wang and D. J. Zhang, "A Review on the DualFactor Model of Mental Health and Its Prospect,” Chinese Journal of Special Education, No. 10, 2011, pp. 68-73.

[13] World Health Organization, "Promoting Mental Health: Concepts, Emerging Evidence, Practice,” World Health Organization, Geneva, 2005.

[14] D. J. Zhang and X. Q. Wang, “An Analysis of the Relationship between Mental Health and Psychological Suzhi: From Perspective of Connotation and Structure,” Journal of Southwest University, Vol. 38, No. 3, 2012, pp. 69-74.

[15] H. Z. Zhang, "Mental Health Can Not Be Improved through Education," Forum on Contemporary Education, No. 1, 2008, pp. 56-59.

[16] D. L. Shen and H. X. Ma, “On Mental Health Diathesis,” Studies of Psychology and Behavior, Vol. 2, No. 4, 2004, pp. 567-571.

[17] D. J. Zhang, “On School Psychological Suzhi Education,” Southwest China Normal University Press, Chongqing, 2004.

[18] C. Peterson, N. Park and M. E. P. Seligman, "Greater Strengths of Character and Recovery from Illness," The Journal of Positive Psychology, Vol. 1, No. 1, 2006, pp. 17-26. doi:10.1080/17439760500372739

[19] K. W. Merrell, "Better Methods, Better Solutions: Developments in School-Based Behavioral Assessment," School Psychology Review, Vol. 39, No. 3, 2010, pp. 422426.

[20] D. J. Zhang, J. L. Wang, X. H. Li and X. L. Feng, "Educational Psychology in China," Zeitschrift für Pädagogische Psychologie, Vol. 26, No. 1, 2012, pp. 57-67. doi:10.1024/1010-0652/a000060

[21] D. J. Zhang, "Psychological Suzhi and Its Structure,” In: A. M. Columbus, Ed., Advances in Psychology Research, Nova Publication, New York, 2010, pp. 239-250.

[22] D. J. Zhang, J. L. Wang and L. Yu, "Introduction,” In: D. J. Zhang, J. L. Wang and L. Yu, Eds., Methods and Implementary Strategies on Cultivating Students's Psychological Suzhi, Nova Science Publishers, New York, 2011.

[23] C. X. Liu, "A Study of the Implicit Theory on Middle School Student's Psychological Suzh,” Master Thesis, Southwest University, Chongqing, 2011.

[24] D. J. Zhang, Z. Z. Feng, C. Guo and X. Chen, "Problems on Research of Students' Mental Quality," Journal of 
Southwest China Normal University, Vol. 26, No. 3, 2000, pp. 56-62.

[25] D. J. Zhang, “On Man's Mental Quality,” Studies of Psychology and Behavior, Vol. 1, No. 2, 2003, pp. 143-146.

[26] D. J. Zhang, "School Psychological Suzhi Education and Students' Mental Health,” In: D. J. Zhang, C. Guo and L. Yu, Eds., Education Psychology, People's Education Press, Beijing, 2005, pp. 493-525.

[27] Y. M. Ye and X. Y. Dai, "Development of Social Support Scale for University Students," Chinese Journal of Clinical Psychology, Vol. 16, No. 5, 2008, pp. 456-458.

[28] Q. Wang, "Constitutional Theory in Traditional Chinese Medicine,” People’s Medical Publishing Press, Beijing, 2005, pp. 9-15.

[29] Q. Wang, "Status and Prospect of Constitutional Theory in Traditional Chinese Medicine," Chinese Journal of Basic Medicine in Traditional Chinese Medicine, Vol. 8, No. 2, 2002, pp. 6-15.

[30] W. B. He and L. He, "Yellow Emperor's Canon of Medicine and Ecological Medicine in Modern Bio-Psychological Society," Journal of Nanjing University of TCM, Vol. 7, No. 2, 2006, pp. 69-70.

[31] D. M. Davydov, R. Stewart, K. Ritchie and I. Chaudieu, "Resilience and Mental Health," Clinical Psychology Review, Vol. 30, 2010, pp. 479-495.

\section{doi:10.1016/j.cpr.2010.03.003}

[32] K. M. Connor and T. Davidson Jr., "Development of a New Resilience Scale: The Connor-Davidson Resilience Scale (CD-RISC),” Depression \& Anxiety, Vol. 18, No. 2, 2003, pp. 76-83. doi:10.1002/da.10113

[33] Y. Q. Hu and Y. Q. Gan, "Development and Psychometric Validity of the Resilience Scale for Chinese Adolescents,” Acta Psychologica Sinica, Vol. 40, No. 8, 2008, pp. 902-912.

[34] X. Q. Wang, H. C. Li and X. J. Pang, “Analysis on Resilience and Other Factors of the Male Prisoners Who Reenter the Prison," Journal of Wuhan Technical College of Communications, Vol. 12, No. 3, 2010, pp. 54-57.

[35] X. Q. Wang and D. J. Zhang, “The Change of Junior Middle School Students' Life Satisfaction and the Prospective Effect of Resilience: A Two Year Longitudinal Study," Psychological Development and Education, Vol. 28, No. 1, 2012, pp. 91-98.

[36] H. H. Yan, "Mental Health and Mental Health Evaluation Standard,” Education Exploration, No. 7, 2001, pp. 6566.

[37] C. D. Lin, “The Key of Mental Health Education Is to Enhances Students’ Psychological Quality,” The Teaching of Thought and Political Study, No. 11, 2000, pp. 34-35. 